\title{
Educación sexual con enfoque de género en el currículo de la educación obligatoria en España: avances y situación actual
}

\section{Gender-focused sex education in the curriculum of compulsory education in Spain: Advances and state of the art}

\author{
Soraya Calvo González ${ }^{1}$ \\ calvosoraya@uniovi.es \\ Universidad de Oviedo, España
}

\section{Resumen:}

El recorrido seguido a partir de las transformaciones del currículum y los cambios legislativos sucedidos en el contexto español no parecen haber dado respuesta a las demandas que organizaciones clave como la OMS o la UNESCO han pronunciado en torno al concepto de educación sexual. Este artículo busca adentrarse en la realidad pasada y presente del tratamiento de aspectos como el cuerpo, las emociones, las relaciones interpersonales y las cuestiones ligadas a la igualdad entre sexos y al género en educación obligatoria de nuestro país. Para ello se lleva a cabo un procedimiento de revisión de la legislación educativa que parte de la LGE de 1970 y llega hasta la actual LOMCE y en el que se desgranan las aportaciones y evidencias detectadas en torno a este tema. Así mismo, el artículo pretende dibujar el recorrido del marco académico de la sexología y la educación sexual como ámbitos del conocimiento, resaltando las tendencias más significativas. Final-

\begin{abstract}
:
The curricular transformations and the legislative changes occurred in the Spanish context do not seem to have addressed the demands that key organizations such as OMS or UNESCO have made about sex education. In the specific context of Spanish Secondary Compulsory Education, the present article taps into the past and present of concepts such as the body, emotions, interpersonal relationships and issues linked to equality and gender. To this end, we conducted a review of the educational legislation from the LGE of 1970 to the current LOMCE with a view to tapping into the most important implications of these legislative frameworks. This article also aims to picture the reality of the academic framework of sexology and sex education looked at as areas of knowledge, highlighting the most significant trends. Finally, we map out the current situation of sexual education as a theoretical body in secondary education. We also highlight the keys which might encourage the incor-
\end{abstract}

1 Dirección para correspondencia (correspondence address):

Soraya Calvo González. Departamento de Ciencias de la Educación. Área de Didáctica y Organización Escolar. Facultad de Formación del Profesorado y Educación. C/ Aniceto Sela s/n, 33005, despacho 014. Oviedo, Asturias (España). 
Educación sexual con enfoque de género en el currículo de la educación obligatoria en España: avances y situación actual

Soraya Calvo González

mente, las conclusiones pretenden dibujar la situación actual de la educación sexual como cuerpo teórico en la etapa secundaria y señalar claves necesarias para que tales contenidos sean incorporados en el currículo educativo y en la formación continua e inicial de profesionales de la educación.

\section{Palabras clave:}

Educación sexual; legislación educativa; género; educación obligatoria; educación para la salud; educación secundaria; juventud. poration of sex education in the educational curriculum and in the initial and continuous training programs for education professionals.

\section{Keywords:}

Sex education; education policy; gender; compulsory education; health education; youth.

\section{Résumé :}

Le parcours qui a suivi les transformations du «Currículum » et les changements législatifs survenus dans le contexte espagnol ne semblent pas avoir répondu aux demandes formulées par des organisations clés telles que l'OMS ou I'UNESCO concernant le concept d'éducation sexuelle. Cet article cherche à plonger dans la réalité passée et présente du traitement d'aspects tels que le corps, les émotions, les relations interpersonnelles et les questions liées au genre et à l'égalité des sexes dans l'enseignement obligatoire dans notre pays. Pour cela, une procédure de révision de la législation éducative est menée commençant à partir de la LGE de 1970 et atteingnant l'actuelle LOMCE, procédure dans laquelle les contributions et les preuves détectées sur cette question sont rassemblées. De plus, I'article vise à tracer le chemin du cadre académique de la sexologie et de l'éducation sexuelle en tant que domaines de connaissance, en mettant en évidence les tendances les plus significatives. Enfin, les conclusions visent à dresser un schéma de la situation actuelle de l'éducation sexuelle en tant que corps théorique au niveau secondaire et à mettre en évidence les clés nécessaires pour que ces contenus soient intégrés dans le programme éducatif et dans la formation continue et initiale des professionnels de l'éducation.

\section{Mots clés:}

Éducation sexuelle, législation éducative, genre, éducation obligatoire, éducation sanitaire, enseignement secondaire, jeunesse.

Fecha de recepción: 07-04-2017

Fecha de aceptación: 18-01-2018

\section{Introducción}

Existe un amplio número de agentes, instituciones públicas para la salud, la educación y el desarrollo; organizaciones no gubernamentales centradas en el trabajo con menores y jóvenes, asociaciones y agrupaciones socioculturales de todo tipo, etc. que han aportado líneas conceptuales a la definición del amplio concepto de educación sexual o educación 
para los sexos, que hoy en día sigue sin asumirse de manera global por una ciudadanía que la mira desde supuestos más morales e ideológicos que académicos. A pesar de ello existe una producción académica relevante que dota de cuerpo teórico a esta disciplina que suele considerarse multidimensional.

Este artículo nace del proceso de revisión bibliográfica y legislativa llevado a cabo en el marco de la tesis doctoral "Identidades (no solo) digitales: aproximación al modelo de comunicación afectiva en redes sociales de la juventud asturiana. Implicaciones para la intervención socioeducativa". En este proceso de investigación surgió la necesidad de conocer en profundidad la situación de la educación sexual con un enfoque de género en el sistema educativo español. Esta tesis doctoral, que tuvo como objetivo primordial generar propuestas reales de intervención educativa en entornos formales y no formales, estudió las construcciones identitarias y las interacciones afectivas de 528 personas jóvenes a través de sus perfiles en redes sociales, poniendo el foco en aquellas cuestiones relacionadas con los conceptos de amor y pareja, los matices de género y la expresión de los propios yoes.

\section{Marco teórico}

\section{La sexología como ámbito formal de conocimiento}

La sexología moderna y su scientia sexualis (Kring, 2010) es muy reciente. Las primeras reacciones académicas a la sexología datan de finales del S.XIX con la conocida obra de Freud, y el desarrollo de las mismas a principios del S. XX fue fuertemente limitado por el ascenso nazi debido a que sus focos de conocimiento iniciales fueron Alemania y Austria. En concreto, cabría destacar la obra de Magnus Hirschfeld, fundador del primer Instituto de Sexología del mundo, que fue saqueado por los nazis en 1933. Posteriormente, las figuras que retomaron el estudio del saber sexológico fueron generando un nuevo entorno de investigación y acción cada vez más alejado de las ideas puramente médicas e incorporando una visión más sociológica del problema de estudio.

Fue Alfred C. Kinsey quien sentó las bases teóricas de la llamada revolución sexual gracias a su Informe Kinsey de dos tomos, uno orientado a la respuesta sexual del hombre y el otro a la de la mujer, y que 
Educación sexual con enfoque de género en el currículo de la educación obligatoria en España: avances y situación actual

Soraya Calvo González

fueron publicados originalmente en 1948 y 1953 (Bullough, 1998). Esta obra supuso para una tendencia heterocentrista y centrada en el hombre un nuevo marco desde el que comprender la sexualidad, incluyendo a la mujer, al colectivo homosexual y a las prácticas fuera del coito. Una década después Virginia E. Johnson y William Masters publicaron una de las referencias modernas en sexualidad más importantes para el avance del Hecho Sexual Humano tal y como lo conocemos hoy en día (Masters y Johnson, 1966). A partir de ese momento la sexología se popularizó como saber en la academia, y comenzaron a adoptarse principalmente formatos para terapia de pareja e investigación en un marco complejo en el que las ciencias de la salud se disputan el dominio de la sexología como ente autónomo (Kring, 2010).

La primera vez que la Organización Mundial de la Salud se refiere a la sexología como rama del saber fue en la década de los 70. Con la publicación de su informe sobre educación y tratamiento de la sexualidad humana la organización buscaba responder a las necesidades de un momento de cambio en el concepto de bienestar social y ciudadano. Estas demandas apuntaban la urgencia de potenciar la figura de una persona especializada en sexualidad desde un enfoque multidisciplinar alejado del puramente diagnóstico, en el que además del rol de terapeuta se incluyera el rol de educador. Así, la OMS defiende su definición de salud sexual como la integración de aspectos somáticos, afectivos e intelectuales del ser sexuado, de modo tal que de ella derive el enriquecimiento y el desarrollo de la persona humana, la comunicación y el amor (OMS, 1974).

En España el Hecho Sexual Humano como ciencia (Amezúa, 2001; Valero-Oltra y López-Gosálbez, 2012) asume en estos momentos una nueva tendencia académica debido a la incorporación moderada y paulatina de las enseñanzas en ciencias sexológicas (Lanas-Lecuona, 1996) a los estudios universitarios de posgrado en diferentes universidades públicas y privadas del territorio nacional.

\section{Discusiones en torno al abordaje educativo del conocimiento sexológico}

Las formas en que los procesos de enseñanza - aprendizaje en torno a los conocimientos generados por la sexología se han ido estructurando y desarrollando requiere un acercamiento progresivo y profundo. Para 
la UNESCO (2010), la educación sexual incorpora un enfoque culturalmente relevante y apropiado a la edad del participante, que enseña sobre el sexo y las relaciones interpersonales a través del uso de información científicamente rigurosa, realista y sin prejuicios de valor. La educación en sexualidad brinda a la persona la oportunidad de explorar sus propios valores y actitudes y desarrollar habilidades de comunicación, toma de decisiones y reducción de riesgos respecto de muchos aspectos de la sexualidad. A pesar de esta defensa que implica un anclaje eminentemente pedagógico, las primeras iniciativas de programas y formatos de educación sexual desde el punto de vista más internacional, así como sus análisis y evaluaciones, fueron fundamentadas principalmente desde las ciencias de la salud. Esta tendencia focaliza el trabajo en torno a conceptos orientados a la prevención de embarazos no deseados e infecciones de transmisión genital; priorizando contenidos centrados en las primeras relaciones heterosexuales con penetración (Fernández et al., 2000; Luker, 2006). Esta realidad ha sentado un precedente importante que no podemos dejar de abordar: se obvian las primeras indicaciones de la OMS, lo que mantiene la medicalización de los contenidos de la educación sexual. Esta particularidad es muy evidente en España, y ha derivado en una merma considerable de la influencia de las teorías de la educación, y de las ciencias sociales en general, en el cuerpo teórico de la sexología, Ilegando a plantear "una sanitarización de la sexualidad que no resuelve el déficit pedagógico" (Rubio-Gil, Martin-Pérez, MesaOlea, y Mesa-Olea, 2008, p. 15)

La educación sexual es un ámbito de actuación didáctica que presenta múltiples peculiaridades en función del entorno sociopolítico en el que se desarrolle. La cultura educativa, la situación ideológica del país, o incluso la tradición moral y la vinculación de las diferentes religiones al sistema educativo estatal han influido, históricamente, sobre la perspectiva desde la que se trabajan en el aula los conceptos relacionados con el cuerpo, las emociones, la expresión erótica y las relaciones de pareja. Estos aspectos fueron también decisivos en la implementación o no de este tipo de intervenciones, por lo que en muchos casos la progresión de iniciativas de educación sexual fueron limitadas y/o promovidas por intereses alejados de los puramente educativas. Uno de esos intereses es el que tiene que ver con la concepción de la sexualidad que la iglesia católica en su representación más popular ha venido defendiendo sin apenas variación en la época moderna (Araujo, 2005). 
Educación sexual con enfoque de género en el currículo de la educación obligatoria en España: avances y situación actual

Soraya Calvo González

La UNESCO (2010) considera prioritaria la educación sexual, entendiendo que el acceso a información y orientación en esta materia es un derecho humano irrenunciable y en favor del cual todos los agentes sociales, políticos y educativos deben desplegar esfuerzos e intereses. En línea con estas recomendaciones, un grupo de trabajo de personas expertas, conformado por miembros de organizaciones internacionales y estatales, desarrollaron en el año 2011 el documento "Educación para la sexualidad con bases científicas" (VVAA, 2011) con el objetivo de servir de guía, apoyo "y proponer estrategias y acciones concretas y consensuadas, encaminadas a implantar una educación sexual con bases científicas para lograr el ejercicio pleno de los derechos sexuales" (VVAA, 2011, p. 4). Entre las instituciones colaboradoras se encuentran referencias estatales como las Universidades de Salamanca y de Vigo e internacionales como las Universidades de Alberta (Canadá), Minnesota (Estados Unidos) o la Pedagógica Nacional de México, además de entidades relevantes tales como la Asociación Mundial para la Salud Sexual (WAS), la Federación Internacional de Planificación Familiar (IPPF) o las ya nombradas UNESCO y OMS. En este documento se explicitan algunas de las conclusiones derivadas del Encuentro Mundial de Expertos en Educación Sexual, Ilevado a cabo en el marco del XV Congreso Latinoamericano de Sexología y Educación Sexual (CLASES).

Para conseguir una educación sexual basada en evidencias científicas se propone una estrategia que parte de una perspectiva de salud pública y justicia social, con obligatoriedad por parte del estado de incluir esta disciplina de manera formal y no transversal en el currículo educativo, garantizando la inversión necesaria para su implementación y posterior evaluación. La estrategia debe incluir una colaboración bidireccional entre sistema educativo y de salud, y estar sometida al cumplimiento del marco jurídico internacional de los derechos humanos. Este grupo de expertos y expertas defiende que la formación de profesionales en educación sexual es imprescindible para dotar de rigor este proyecto, del cual debe ser participe la comunidad educativa por completo, desde el profesorado hasta las familias y el alumnado. Para lograr los objetivos la implicación de los medios de comunicación es fundamental en tanto que son considerados agentes educativos de gran magnitud, y su labor divulgadora se convierte en altavoz social del trabajo en red que instituciones, sociedad civil, organizaciones no gubernamentales y agentes comunitarios Ilevan a cabo. Por último, señalar que se proponen accio- 
nes particulares para formar a colectivos específicos que abarquen la variabilidad humana más extensa y diversa.

\section{La educación sexual como derecho: dificultades para la puesta en marcha de propuestas didácticas significativas}

A pesar de la formulación de documentos y proyectos articulados con el apoyo de entidades internacionales de prestigio, cuyas intenciones fueron eminentemente prácticas y con opciones para favorecer la progresividad de su implantación, la situación de la educación sexual no ha sufrido modificaciones sustanciales. Numerosas organizaciones han formulado denuncias públicas debido a esta realidad sostenida: el Consejo de Juventud de España, la Sociedad Española de Enfermedades Infecciosas, la Federación de Planificación Familiar Estatal y la Sociedad Española de Contracepción demandan planes de educación sexual con personal cualificado en sexología, y se aboga por la creación de centros de referencia de información y educación sexual. En lo que a salud sexual se refiere, el estudio Barómetro del acceso de las mujeres a la libre elección de anticonceptivos modernos en 10 países de la UE (IPPF, 2013) denuncia la situación de España y propone varias medidas de actuación en educación sexual, que incluyen, entre otras, "garantizar una educación sexual obligatoria que cuente con el tiempo y los recursos suficientes" o "desarrollo de un marco de referencia orientativo para el profesorado y una oferta de formación adecuada para la enseñanza de la educación sexual, que incluya el abordaje de la diversidad cultural" (IPPF, 2013, pp. 4-5)

Otros estudios (Alfaro, Vázquez, Muñoz, Rodríguez, y González, 2015) profundizan en la cuestión sobre la información sexual consumida y arrojan datos en torno a la eminente importancia de los medios de comunicación como agentes informadores. Las Tecnologías de la Información y la Comunicación actúan como altavoces ya que los contenidos encontrados a través de las redes pueden ser divulgados entre iguales, favoreciendo el intercambio ágil y accesible de mitos y estereotipos (Alfaro et al., 2015). Estas conclusiones parecen confirmar que la educación sexual recibida en los centros educativos no es especialmente relevante para sus concepciones personales y colectivas en torno a la sexualidad y que el acceso a informaciones mediáticas sin una dinamización por profesionales de la sexología conlleva acceder a contenidos con datos 
Educación sexual con enfoque de género en el currículo de la educación obligatoria en España: avances y situación actual

Soraya Calvo González

erróneos o falseados, mitos, tendencias no centradas en la igualdad y coeducación, e influencias del porno más comercial. Todo ello deriva en una tendencia a la indefensión a la hora de hablar sobre sexualidad como aspecto ligado a la vida sin miedos ni temores (Eresta y Delpino, 2013). Y a pesar de todo ello, en este entorno de intoxicación "la mayoría de jóvenes se consideran bien o muy bien informados en materia de sexualidad" (Alfaro et al., 2015, p. 223).

Finalmente, y en íntima relación con todo el desarrollo anterior, destacamos la falta de formación inicial universitaria (Martínez et al., 2013) y continua en nuestro país sobre educación sexual orientada a profesionales de la educación en activo o incorporada en los planes de estudio de magisterio, pedagogía o en los máster de formación del profesorado, lo que desencadena un estado general de carencia formativa. Es también evidente una dificultad derivada de la existencia de discursos de determinados profesionales contrarios a la incorporación de proyectos de igualdad y coeducación (Rebollo Catalán, Vega Caro, y García Pérez, 2011). Si bien se ha instado desde la administración a incluir la educación sexual como materia transversal (como veremos en el posterior análisis legislativo que integra este artículo), en la práctica no se ha garantizado la formación inicial del profesorado (Martínez et al., 2011, 2013, 2014) siendo demandado por el colectivo mayor compromiso e implicación por parte del sistema público (García-Vázquez, J., Ordóñez, A. y Arias-Magadán, S., 2014). Destacar el escaso número de publicaciones académicas vinculadas a estas cuestiones que presenten resultados en el contexto del Estado Español.

\section{Análisis legislativo del problema de estudio}

El siguiente apartado de este artículo trata de revisar los cambios y posibles avances que las diferentes leyes educativas desarrolladas en España han ido incorporando en torno a la educación sexual con los siguientes objetivos:

- Desgranar los cambios acontecidos en torno a la incorporación de la educación sexual con perspectiva de género como cuerpo teórico en educación secundaria en las diferentes leyes educativas desde la transición hasta la actualidad.

- Conocer la manera en que la educación sexual con perspectiva de 
género se incorpora en el marco formal de la actual ley educativa (LOMCE); valorando esta incorporación en base al recorrido legislativo previo.

- Señalar claves necesarias a tener en cuenta para la implantación de una educación sexual con perspectiva de género en el currículo de la etapa secundaria obligatoria.

Para comenzar a analizar la situación en un marco político de estabilidad, y situándonos en los últimos años de la dictadura franquista, debemos remontarnos a la Ley General de Educación, también conocida por sus siglas LGE (Ley 14/1970, de 4 de agosto, General de Educación y Financiamiento de la Reforma Educativa) ley educativa que surgió en plena época de la transición y que fue implantada en los centros educativos de todo el territorio nacional a partir del año 1970.

\section{La Ley General de Educación de 1970 (y su convivencia con la Ley Orgánica de Derecho a la Educación)}

En el marco teórico de esta ley, y en relación con la educación sexual, solo se introducen en el currículo educativo aspectos vinculados de manera directa con el plano reproductivo de la sexualidad, siendo en muchos casos tratado en exclusiva desde el conocimiento anatómico. Tanto en educación general básica como en el Bachiller Unificado Polivalente el trabajo con valores, actitudes y habilidades estaba relegado en pro de la importancia de los métodos de enseñanza academicistas. Es, por tanto, que la educación sexual como concepto explícito en esta época no se planteaba, ni se estructuraba formalmente dentro del currículo educativo.

Como aspectos más Ilamativos de la Ley General de Educación, destacamos cómo las enseñanzas mínimas en las etapas de la EGB, única etapa educativa obligatoria, en cuanto a su relación con una educación sexual desde una perspectiva global fueron exclusivamente orientadas a la noción teórica del cuerpo, incluyendo el conocimiento y la práctica de medidas de higiene del aparato reproductor y cuidados necesarios de la madre durante la gestación, parto y lactancia en el Real Decreto 3087/1982, de 12 de noviembre.

En el desarrollo del Capítulo 11 de la Ley 14/1970, se enmarca el 
Educación sexual con enfoque de género en el currículo de la educación obligatoria en España: avances y situación actual

Soraya Calvo González

artículo 17 de la sección segunda, destinada a tratar cuestiones propiamente relativas al nivel EGB. En este artículo se remarca que los programas y orientaciones pedagógicas serán establecidos por el Ministerio de Educación y Ciencia con la flexibilidad suficiente para poder ser matizados de acuerdo con el sexo. Tal aportación deja entrever que la educación podría estar diferenciada según el sexo del alumno o de la alumna, premisa vinculada a la corriente de la segregación educativa por sexos. Esta premisa entró en conflicto con el ideario educativo constitucional (Aláez, 2009) correspondiente a la Constitución Española de 1978, que si bien fue promulgada años después del inicio de la LGE, convivieron legislativamente durante más de una década. Ese ideario educativo del que se nutre la Constitución Española incluye entre los fines últimos a los que debe servir la educación "la transmisión del valor de la igualdad entre hombres y mujeres y la prohibición de discriminación por razón de sexo" (Aláez, 2009, p. 41) En este momento es necesario repensar si una ideología como la que se sitúa desde la perspectiva de educación diferenciada, basada en una organización de la enseñanza a partir de diferencias psicológicas, biológicas, etc. derivadas del sexo, "permite (debido a la metodología de segregación por el género que implica) una garantía del derecho a la educación conforme al ideario educativo constitucional" (Aláez, 2009, p. 42).

La LODE, Ley Orgánica de Derecho a la Educación (Ley Orgánica 8/1985, de 3 de julio, reguladora del Derecho a la Educación), que convivió durante 5 años con la Ley General de Educación, retoma en su artículo 32 la necesidad de garantizar una igualdad de derechos y oportunidades de todos los alumnos y todas las alumnas, desplazando la discriminación por sexo y favoreciendo una educación comprensiva y de equidad.

\section{La Ley de Ordenación General del Sistema Educativo de 1990: transversalidad, igualdad de oportunidades y educación para la salud}

La Ley General de Educación fue derogada en el año 1990, momento en el cual aparece en el marco político la LOGSE (Ley Orgánica 1/1990, de 3 de octubre, de Ordenación General del Sistema Educativo). Esta ley expande el periodo de escolarización obligatoria, que va desde los 6 a 
los 16 años en dos etapas educativas (educación primaria y educación secundaria obligatoria), y que supuso una apuesta política de renovación socioeducativa que pretendía introducir características relevantes que marcaran un antes y un después en la manera de entender la educación pública en España. Una de los aspectos a destacar de la LOGSE es el concepto de contenido transversal. La transversalidad de un contenido supone una intencionalidad de trabajo activo y global desde las distintas materias que conforman el Plan de Estudios de cada curso, lo que en la práctica terminó significando una presencia sobre el papel de contenidos que, finalmente, no se concretan de manera real en el día a día del alumnado. La intención de formar parte del espíritu del proceso educativo de esta transversalidad tuvo escasa traducción en el día a día docente (Esteban-Frades, 1996).

En el marco de esa transversalidad la perspectiva de género adquiere protagonismo. Ya en el preámbulo de la ley se reafirma la importancia de la educación para avanzar en la lucha contra las desigualdades, señalando específicamente aquellas derivadas del sistema sexo-género. Los Reales Decretos que regulan los mínimos de Educación Infantil, Primaria y Secundaria en el marco de la LOGSE señalan claramente aspectos como la necesidad de trabajar en pro de la revalorización de los cuidados, el valor de las diferencias, el análisis del lenguaje sexista o el reconocimiento del feminismo como proyecto moderno de valor ético (Madrid y Lucer, 2010). Junto a ello, se instaura la materia transversal "Educación para la lgualdad de Oportunidades" y la proyección del modelo de escuela mixta como una buena práctica co-educativa (Bejarano y Mateos, 2014). Este un potencial avance a diferentes niveles: teórico, pues se fomentó la deconstrucción de preconceptos negativos para la igualdad, pero socialmente aceptados; político, al proponer e instaurar un nuevo modelo de escuela y pautas legales desde una educación holística y de justicia social; y de práctica docente.

En la LOGSE la educación sexual es asumida también como un contenido transversal dentro del bloque de "Educación para la Salud", compendio de contenidos teóricos, procedimentales y actitudinales que partía de una idea holística de salud que implica ampliar lo que tradicionalmente se conoce como salud "para acercarnos a su dimensión comunitaria, que debe hacer referencia a las mejoras del nivel de salud de la misma con la participación activa de la población y desde las propias bases cognitivas y experienciales, esto es, antropológicas" (García-Mar- 
Educación sexual con enfoque de género en el currículo de la educación obligatoria en España: avances y situación actual

tinez y Benito-Martinez, 1996, p. 138). A pesar de su aparente relación directa con este bloque, la educación sexual y sus principios, tal y como los hemos presentado a lo largo de este estudio, también presentaban profundas vinculaciones con los bloques transversales de Coeducación y Educación moral, para la convivencia y la paz. Los bloques de asignaturas transversales comprendían un conjunto de conocimientos y habilidades a adquirir a lo largo de los diferentes cursos educativos que conformaban cada etapa. Estos contenidos de bloque estaban muy ligados con los objetivos generales y contenidos mínimos previstos para cada etapa educativa, que en el caso de la LOGSE comenzaban a vincularse con la necesidad de trabajar una educación sexual más allá de la cuestión genital. Además de mantener las ideas sobre el conocimiento del cuerpo, se incorpora, por primera vez, el concepto de relación afectiva y sexual dentro de las enseñanzas mínimas de educación primaria, así como la valoración de aspectos sociales y culturales de la sexualidad (Real Decreto 1006/1991 sobre Enseñanzas mínimas de la Educación Primaria).

Durante la etapa de educación secundaria obligatoria se incorporaron contenidos que aludían a la sexualidad humana como comunicación afectiva y opción personal, atendiendo a las diferentes pautas de conducta sexual. Para esa misma etapa y sumándose a los aspectos relacionados con los hábitos de higiene sexual y anticoncepción, la LOGSE introduce explícitamente la idea de la discriminación sexual y la necesidad de trabajar desde una perspectiva de respeto y diversidad, lo que parece construir un marco legislativo más acorde con el ideario educativo constitucional que la ley educativa predecesora.

\section{Los pioneros de la educación sexual: El Colectivo Harimaguada}

Una de las propuestas más ambiciosas de la LOGSE fue la de abrir y descentralizar el currículo (Viñao, 1994) permitiendo que las Comunidades Autónomas y los centros educativos fueran capaces de adaptar la legislación nacional a las realidades contextuales. El cuerpo teórico del desarrollo de los aspectos didácticos de esta ley expone la idea de autonomía pedagógica, lo que parece favorecer la inclusión en las programaciones curriculares de los centros de nuevas propuestas en colaboración con organizaciones e instituciones externas que dieran respuestas a las ne- 
cesidades educativas de cada centro en concreto. Por tanto, se formula una nueva metodología de base en la cual se hace visible la tendencia hacia la vinculación entre el marco educativo formal y la sociedad. Para la educación sexual esto supuso un nuevo marco de posibilidades que se materializó de diversas formas, siendo la más reseñable la experiencia del Colectivo Harimaguada (Díaz-Acosta, 2010) con propuestas para profesorado y centros.

A pesar de la repercusión que el Colectivo Harimaguada tuvo en todo el país, la tendencia de plasmar esa autonomía pedagógica no consiguió transformar una realidad educativa a nivel global. Por todo ello, la incorporación en profundidad de aspectos relacionados con la educación sexual que no se quedaran en el cumplimiento de los contenidos mínimos fue totalmente determinado por la iniciativa y los recursos que cada centro dispusiera de manera individual, y no siempre esa disposición o intención autónoma garantizaba una apuesta posible en la práctica.

\section{La Ley Orgánica de Educación del 2006 y los debates en torno a Educación Para La Ciudadanía}

Aunque se desarrolló una nueva ley Ilamada Ley Orgánica de Calidad de la Educación (Ley Orgánica 10/2002, de 23 de diciembre, de Calidad de la Educación) que modificaba sustancialmente los contenidos del currículo educativo, esta ley nunca llegó a materializarse. Por tanto, la ley que sucede a la LOGSE en términos de realidad educativa fue la Ley Orgánica de Educación (Ley Orgánica 2/2006, de 3 de mayo, de Educación). Ya en su preámbulo se incorpora el término de educación sexual, relacionándose de manera interdisciplinar con una visible influencia de la Ley contra la Violencia de Género. Entre los fines de la educación se resalta el pleno desarrollo de la personalidad y de las capacidades afectivas del alumnado, la formación en el respeto de los derechos y libertades fundamentales y de la igualdad efectiva de oportunidades entre hombres y mujeres, el reconocimiento de la diversidad afectivo-sexual, así como la valoración crítica de las desigualdades, que permita superar los comportamientos sexistas.

La educación afectivo-sexual se convierte en una necesidad y una finalidad explicitada, aunque aún no se plantea el diseño de una manera específica y se mantiene como transversal desde el gran tema 
Educación sexual con enfoque de género en el currículo de la educación obligatoria en España: avances y situación actual

Soraya Calvo González

nombrado como educación para la salud y educación sexual (Jurado, 2009).

La LOE continuó con el espíritu abierto que tenía la LOGSE. Se dio continuidad al trabajo en relación con organizaciones implicadas en la sociedad y el contexto cercano, permitiendo que cada centro generara proyectos y propuestas autónomas, entre las que se encontraban algunas relacionadas con la educación sexual. Sin embargo, estas propuestas no siempre fueron llevadas a cabo por personal formado en sexología, ya que no existe una regulación específica que limite el acceso al campo de la educación sexual de profesionales no capacitados profesionalmente, siendo común la gestión de la educación sexual externa por parte de personal voluntario, personal de ocio y tiempo libre, o titulados en ramas dispares del conocimiento. Por otro lado, no existe tampoco uniformidad en torno a estos programas, por lo que dependen en exclusiva del interés de cada centro y de los recursos disponibles, de la misma manera que sucedía en la anterior ley.

En cuanto a su descentralización y derivación de las competencias educativas a las comunidades autónomas, esto pudo reseñarse especialmente en el desarrollo documental y educativo de las Ilamadas Competencias Básicas (Tiana, 2011) generalmente definidas por ocho ítems. En torno a este esqueleto de competencias básicas se estructuran los objetivos generales de etapa, núcleo fundamental del desarrollo competencial, ya que dieron forma a las asignaturas y los contenidos mínimos que a nivel nacional se establecen por parte de Ministerio de Educación. En cuando a los objetivos generales, también existen novedades que posicionan a la LOE en un espacio más proclive al trabajo en educación sexual. En los objetivos generales de cada etapa educativa obligatoria, las cuales siguen la misma estructura que la LOGSE, se aprecia una apuesta por reflejar la necesidad de trabajar en el proceso de enseñanza con los cuerpos, las capacidades afectivas o el valor y el respeto entre los sexos.

En base a estos objetivos podemos diferenciar entre diferentes contenidos conceptuales, actitudinales y procedimentales que irán desplegados en la planificación de cada asignatura obligatoria y/u optativa. En general, aquellos contenidos más relacionados con una educación sexual para la salud, como las cuestiones fisiológicas o los procedimientos de higiene, eran impartidos en las asignaturas de Educación Física y Conocimiento del Medio en Educación Primaria, o Ciencias Naturales en Educación Secundaria. Como hándicap, la formación docente en 
educación sexual es aún muy escasa (Martínez et al., 2011) limitando así la perspectiva que favorece una incorporación real de la misma al proceso total de E-A.

Como importante novedad, esta ley incorpora al currículo una nueva asignatura con impartición en Educación Primaria y Educación Secundaria, Ilamada Educación para la Ciudadanía y los Derechos Humanos, estructura formal en la que sí se consideran de manera concreta contenidos relativos a la sexualidad humana y su aspecto más social: nuevos modelos de familia y de relaciones, derechos reproductivos y sexuales, posicionamiento de las identidades y orientaciones, etc.

La asignatura de educación para la ciudadanía, como comúnmente fue conocida, generó polémica en la comunidad académica y educativa, Ilegando esa polémica también a la opinión ciudadana. La manera en que la asignatura proponía cuestiones sobre familia, violencia de género y relaciones diversas fueron algunos de los puntos rojos que organizaciones como el Foro de la Familia o la Confederación Nacional Católica de Padres y Alumnos (CONCAPA) denunciaron de manera formal, llevando a cabo diferentes acciones a favor de una objeción de conciencia. Dicha objeción de conciencia consistía en una negación a cursar la asignatura por parte de padres, madres o alumnado, pretendiendo también que el resultado de la misma computase a efectos de media académica. Este movimiento fue especialmente complejo debido a que dicha asignatura era obligatoria.

Matilde Peinado (2008) recopila algunos de los argumentos centrados en la homosexualidad (o "anti-homosexualidad") que fueron utilizados por estas organizaciones contrarias a la asignatura: fundamentación de la asignatura en la teoría de género, siendo esta teoría un ataque contra el hombre y una herramienta de ideologización; se niega la complementariedad sexual entre hombre y mujer, la cual es la base de la familia tradicional; la homosexualidad es un concepto que trastoca toda la civilización y que pone en peligro la idea de familia tradicional; se muestran en contra de que las orientaciones sexuales deban ser aceptados por todos y todas; hay orientaciones que no son normales ni adecuadas. Otros argumentos esgrimidos aluden a la necesidad de que sean las familias quienes traten la educación sexual en casa, ya que el Estado no tiene la capacidad moral para poder involucrarse en esta labor, o el derecho de los padres a proporcionar una educación heterosexual a sus hijos e hijas. En definitiva, se exige que se eviten cuestiones sobre sexualidad en las 
Educación sexual con enfoque de género en el currículo de la educación obligatoria en España: avances y situación actual

Soraya Calvo González

aulas ya que, según los colectivos denunciantes, éstas pertenecen a la vida privada de las familias. Por último, señalar que la LOE también incluye dentro de su normativa un interesante punto a destacar en torno a la configuración y estructuración de la composición del Consejo Escolar, desarrollada en el artículo 126 del documento marco de la Ley Orgánica 2/2006. El segundo punto del artículo explica que, tras la constitución del Consejo Escolar, éste debe asignar una persona que impulse aquellas medidas educativas necesarias para fomentar la igualdad real y efectiva entre hombres y mujeres. Esta alusión a la necesidad de incorporar medidas de coeducación reales supone sobre el papel un cambio sustancial y significativo, cuya puesta en práctica real ha sido, al menos, incierta.

\section{La Ley Orgánica de Mejora de la Calidad Educativa del 2013: lo que no se nombra no existe}

A la LOE le sucede la Ley Orgánica de Mejora de la Calidad Educativa (LOMCE) en el año 2013. Esta ley marca un antes y un después en el trato educativo que se da los conceptos de identidad, orientación sexual y expresión emocional Con la LOMCE las oportunidades formales orientadas a dedicar tiempo en el aula a la educación de los sexos se reducen debido a la supresión de Educación para la Ciudadanía y los Derechos Humanos y su sustitución por la asignatura Educación Cívica y Constitucional. El cambio en la denominación de la asignatura lleva consigo cambios en los contenidos.

“(...) Se suprimen algunos contenidos recogidos en la declaración universal de derechos humanos, como es el reconocimiento de la diversidad en los modelos familiares y en la orientación sexual. Además, se elimina el estudio de las causas que originan la violación de los derechos humanos, la desigualdad en el planeta y la desigualdad de género" (Prats, 2012, 7-8).

Esta nueva asignatura, en palabras del Ministro de Educación propulsor de la LOMCE, Ignacio Wert, está libre de cuestiones controvertidas (Díaz-Sotero, 2012). Tal transformación de contenidos pone a España en una situación compleja respecto a los países vecinos del entorno europeo por limitar el tratamiento educativo de los derechos de las personas a un constitucionalismo de mirada estrecha (Prats, 2012). 
Las Competencias Básicas trabajadas en la LOE cambian también de denominación, siendo ahora conocidas como Competencias Clave. Este nuevo formato pasa a ser de 7 referencias debido a que se unen las vinculadas a matemáticas con las específicas de ciencias y tecnología. En la LOMCE la educación sexual no se nombra como tal, por lo que los contenidos que pudieran tener relación con ella se mostrarán dispersos y con carencia argumental entre las asignaturas obligatorias.

Respecto a las etapas educativas y sus contenidos específicos, y contemplando siempre la perspectiva del Ministerio de Educación, podemos centrarnos inicialmente en el currículo específico de Educación Primaria plasmado en el Real Decreto 126/2014, de 28 de febrero. En una primera búsqueda de reconocimiento, es reseñable que en el documento no se hace ningún tipo de referencia al concepto sexualidad de manera directa más allá del concepto de abuso sexual y su prevención. Sin embargo, se reafirma que las administraciones educativas deberán asegurar y fomentar el desarrollo de valores que fomenten la igualdad entre hombres y mujeres y la prevención de la violencia de género, así como el trabajo en pro de los valores inherentes al principio de igualdad de trato y no discriminación por cualquier condición personal o social.

Entre los objetivos generales de Educación Primaria encontramos enunciados que abordan las diferencias culturales, la no discriminación de personas con discapacidad, la higiene y la salud, la aceptación del propio cuerpo y el de los otros y el desarrollo de las capacidades afectivas en todos los ámbitos de la personalidad y en sus relaciones con los demás. Se remarcan de manera negativa las actitudes violentas y los prejuicios o estereotipos sexistas.

En cuanto a las asignaturas obligatorias, en el cuerpo de la asignatura Valores Sociales y Cívicos se habla de autoestima, autoconcepto, respeto y empatía de manera recurrente en el Bloque 1. La identidad y la dignidad de la persona y Bloque 2. La comprensión y el respeto en las relaciones interpersonales, sin profundizar en aspectos de emoción, sentimientos o sexualidad. Entre los contenidos específicos de Ciencias de la Naturaleza se trabaja cuerpo e igualdad entre hombres y mujeres, siendo incluidos aspectos sobre cuidado del cuerpo e higiene también en la asignatura de Educación Física.

En el documento de mínimos para la Educación Secundaria Obligatoria, (Real Decreto 1105/2014, de 26 de diciembre) las alusiones a la educación sexual son más claras que en Educación Primaria. Se explici- 
Educación sexual con enfoque de género en el currículo de la educación obligatoria en España: avances y situación actual

Soraya Calvo González

tan conceptos como la tolerancia, la cooperación y la solidaridad desde la igualdad de trato y de oportunidades entre mujeres y hombres; se dan valor a las diferencias entre los sexos y se rechaza la discriminación de las personas por razón de sexo, los estereotipos y las manifestaciones de violencia contra la mujer. Así mismo se incorporan ideas en torno al refuerzo de las capacidades afectivas, los hábitos sociales y el funcionamiento del propio cuerpo. A destacar el objetivo dedicado a valorar la dimensión humana de la sexualidad toda su diversidad.

Al igual que en el caso de Primaria, los contenidos que aluden a la sexualidad se organizan de manera transversal entre las distintas asignaturas que conforman el plan de estudios de los diferentes cursos. En la asignatura Biología y Geología para $1^{\circ}$ y $3^{\circ}$ curso es donde más énfasis se hace en los mismos, siendo La respuesta sexual humana. Sexo y sexualidad. Salud e higiene sexual un contenido incorporado dentro de su Bloque 4. Las personas y la salud. Promoción de la salud. También en Educación Plástica y Visual para $4^{\circ}$ curso se presenta la necesidad de trabajar sobre las necesidades de consumo creadas por la publicidad rechazando los elementos de ésta que impliquen discriminación sexual, social o racial. En la asignatura Valores Éticos para primer ciclo y $4^{\circ}$ de la ESO se habla de las relaciones interpersonales y la igualdad en las mismas, aunque no se alude específicamente a las relaciones íntimas o amorosas. Cabe destacar que esta asignatura es la alternativa a religión, de manera que aquel alumnado que por motivos morales acuda a la asignatura católica no cursará estos contenidos.

\section{Conclusiones}

Tras este análisis legal, que responde al primero de los objetivos señalados en este artículo, nos encontramos con una legislación en la que si bien se mantienen ciertos contenidos transversales que podrían relacionarse con un trabajo holístico en sexualidad humana, carece de espacios formales en el currículo educativo destinados a incorporar la educación sexual como cuerpo teórico propio.

El segundo objetivo del estudio era conocer la manera en que la educación sexual con perspectiva de género se incorpora en el marco formal de la actual ley educativa, valorando esta incorporación en base al recorrido legislativo previo. Al ser la LOMCE una legislación en pro- 
ceso de implantación aún no se puede hacer una valoración final de su efecto sobre la democratización o no de la educación en igualdad y la educación sexual. A pesar de ello sí podemos concluir por todo lo anteriormente expresado que su desarrollo formal no ha seguido las directrices que abogan por una incorporación en el sistema educativo de una educación sexual científica, global, y para todo el alumnado (OMS, 2010; UNESCO, 2010; VVAA, 2011). Respecto a la perspectiva de género, Ferrer y Bosch (2013) hacen una revisión a partir de diferentes estudios empíricos en torno a la materia y llegan a la conclusión de que la educación para la igualdad está aún lejos de ser una verdadera realidad en las aulas. Esto es debido a múltiples factores que superan las formalidades de la legislación educativa, como pueden ser van la transmisión de la cultura hegemónica a través currículo oculto; la tendencia sexista de los libros de texto o las pautas sexistas que determinan el recorrido académico que alumnos y alumnas siguen de manera diferenciada. Estudios más actuales que aluden específicamente a los cambios introducidos a partir de la LOMCE, como el Ilevado a cabo por Fernández-González y González (2015), hablan de un verdadero retroceso respecto a las políticas anteriormente implantadas. En concreto, se defiende que el enfoque de contenidos vinculados a los conceptos de género e igualdad encajan en un modelo aséptico y superficial que es insuficiente para generar cambio social y aprendizajes significativos. En suma con todo lo anteriormente destacado, denunciar que la LOMCE es un constructo eminentemente centralista que reduce a la mínima expresión las competencias educativas de las Comunidades Autónomas. Esta situación se puede entender como un hándicap para las posibilidades que los contextos socioeducativos relevantes tienen para desarrollar proyectos curriculares y/o extracurriculares propios y diversos, lo que podría favorecer la existencia de un clima de desequilibrio y desigualdad que afecte a los centros educativos españoles. El resultado parece claro: existirán proyectos que no llegarán a todos los centros, no habrá coordinación y consenso en los criterios de actuación y no todos los equipos docentes estarán involucrados. La igualdad de oportunidades que promovía la LOGSE como eje central de su proyecto curricular se verá desmerecida.

Por último, y como respuesta al tercero de los objetivos planteados, reafirmarnos en lo oportuno de plantear en el currículo formal contenidos que planteen diálogos, conversaciones y disertaciones que aborden 
Educación sexual con enfoque de género en el currículo de la educación obligatoria en España: avances y situación actual

Soraya Calvo González

cuestiones controvertidas con el fin último de generar verdadera conciencia crítica y favorecer una toma de decisiones efectivas y conscientes. Por todo ello consideramos que el documento "Educación para la sexualidad con bases científicas" (VVAA, 2011) sigue plenamente de actualidad y suscribimos sus puntos y claves. Aportamos a este documento la necesidad de llevar a cabo un trabajo "sex-positivo", alejado de las políticas del miedo y de directrices hegemónicas, como el heterocentrismo y el patriarcado. Junto a ello, la necesidad de que el esfuerzo sea proconvivencial y diverso, lo que requiere un enfoque de ciudadanía activa y participativa que incluya aspectos sobre la gestión de los conflictos intra e interpersonales y sociales (Pineda-Alfonso, 2015). La incorporación de estos contenidos, que por su propia naturaleza superan lo puramente sanitario, parte irrenunciablemente de un compromiso que garantice la formación inicial y continua de los y las docentes (Malón, 2012). Finalmente, creemos firmemente que abordar formal y comprensivamente la educación sexual repercutirá de manera positiva en la lucha contra la violencia de género y las múltiples formas de discriminación, así como en el desarrollo pleno, saludable y consciente de las identidades personales y sexuales. Todo ello contribuirá a la construcción de una sociedad más justa y equitativa.

\section{Referencias}

Aláez, B. (2009). El ideario educativo constitucional como fundamento de la exclusión de la educación diferenciada por razón de sexo de la financiación pública. Revista Española de Derecho Constitucional, 29(86), 31-65.

Alfaro, M., Vázquez, M., Muñoz, M., Rodríguez, L., y González, C. (2015). Hábitos sexuales en los adolescentes de 13 a 18 años. Revista Pediatría Atención Primaria, 17, 217-225.

Amezúa, E. (2001). Educación de los sexos. La letra pequeña de la educación sexual. Revista Española de Sexología, 107-108.

Araujo, K. (2005). Sobre ruidos y nueces: debates chilenos en torno a la sexualidad. Iberoamericana, 8, 109-125.

Bejarano, M. y Mateos, A. (2014) Género y Sexualidad en la formación inicial de maestros y maestras. ¿Por qué no un currículum sexual?. Revista Exedra, número temático Sexualidade, Género e Educaçao, 127-146.

Bullough, V. L. (1998). Alfred Kinsey and the Kinsey report: Historical overview and lasting contributions. Journal of Sex Research, 35(2), 127-131.

Díaz-Acosta, R. (2010). Del papel al multimedia. Los materiales didácticos para la edu- 
Educación sexual con enfoque de género en el currículo de la educación obligatoria en España: avances y situación actual

Soraya Calvo González

cación afectivo-sexual en Graó (Ed.), En M. Area (coord). Materiales y recursos didácticos en contextos comunitarios. 177-183. Barcelona.

Díaz-Sotero, P. (2012). Educación sustituye Ciudadanía por una materia "exenta de cuestiones controvertidas" Periódico El Mundo, 31 de enero del 2012.

Eresta, M. J., y Delpino, M. A. (2013). Relaciones afectivas y sexualidad en la adolescencia. Madrid: Liga Española de la Educación.

Esteban-Frades, S. (1996). Algunas reflexiones sobre los temas transversales en la LOGSE: Limitaciones y posibilidades que tienen los docentes para llevar a cabo una enseñanza en valores. Revista Interuniversitaria de Formación Del Profesorado, (27), 45-59.

Fernández, L., Bustos, L., González, L., Palma, D., Villagrán, J., y Muñoz, S. (2000). Creencias, actitudes y conocimientos en educación sexual. Revista Médica de Chile, 128(6), 574-583.

Fernández-González, N. y González N. (2015). La LOMCE a la luz de la CEDAW. Un análisis de la coeducación en la última reforma educativa. Journal of Supranational Policies of Education, 3, 242-263

Ferrer, V y Bosch, E. (2013) Del amor romántico a la violencia de género. Para una coeducación emocional en la agenda educativa. Profesorado, 17(1),105-122

García-Martinez, A., y Benito-Martinez, J. (1996). La educación para la salud en el contexto de la reforma educativa. Pedagogía Social. Revista Interuniversitaria, 14, 135146.

García-Vázquez, J., Ordóñez, A. L., y Arias-Magadán, S. (2014). Educación sexual: opiniones y propuestas del alumnado y profesorado de los institutos de secundaria de Asturias, España. Global Health Promotion, 21(4), 74-82.

IPPF. (2013). Barómetro del acceso de las mujeres a la libre elección de anticonceptivos modernos en 10 países de la UE. Bruselas: Federación de Planificación Familiar de España.

Jurado, C. (2009). Los temas transversales en la escuela. Innovación Y Experiencias Educativas, 25, 1-11.

Kring, B. (2010). A review of Geschichte der Sexualwissenschaft. Journal of Sex y Marital Therapy, 36(2), 176-179.

Lanas-Lecuona, M. (1996). Sexología: hacia su espistemología interna. Anuario de Sexología, 2, 43-58.

Luker, K. (2006). When sex goes to school: Warring views on sex (and sex education) since the sixties. New York: W. W. Norton.

Madrid, J.M. y Lucer, L.A. (2010). Directrices de la política educativa de género en España (1970-2010). Educatio Siglo XXI, 28(2), 223-244.

Malón, A. (2012). ¿El derecho a la educación sexual? Entre los discursos de salvación y la ausencia de conocimiento. Educatio Siglo XXI, 30(2), 207-228.

Martínez, J. L., González, E., Vicario-Molina, I., Fernández-Fuertes, A. A., Carcedo, R. J., Fuertes, A., y Orgaz, B. (2013). Formación del profesorado en educación sexual: pasado, presente y futuro. Magister, 25(1), 35-42.

Martínez, J. L., Orgaz, B., Vicario-Molina, I., González, E., Carcedo, R. J., FernándezFuertes, A. A., y Fuertes, A. (2011). Educación sexual y formación del profesorado en España: diferencias por sexo, edad, etapa educativa y comunidad autónoma. Revista de Formación Del Profesorado e Investigación Educativa, 24, 37-47. 
Educación sexual con enfoque de género en el currículo de la educación obligatoria en España: avances y situación actual

Soraya Calvo González

Martínez, J. L., Vicario-Molina, I., González, E. y Ilabaca, P. (2014). Sex education in Spain: the relevance of teachers' training and attitudes/Educación sexual en España: importancia de la formación y las actitudes del profesorado. Infancia y Aprendizaje, $37(1), 117-148$.

Masters, W. H., y Johnson, V. E. (1966). Human sexual response. Boston: Little Brown y Co.

OMS. (1974). Education and treatment in human sexuality: the training of health professionals, report of a WHO meeting. Geneva.

OMS. (2010). Estándares de Educación Sexual para Europa. Colonia: OMS.

Peinado, M. (2008). Educación para la ciudadanía y homosexualidad: elementos para un debate. Revista Iberoamericana de Educación, 46(1), 1-10.

Pineda-Alfonso, J. A. (2015). Educar para la ciudadanía trabajando con temas controvertidos en Educación Secundaria Obligatoria. Revista de Investigación Educativa, $33(2), 353$.

Prats, E. (2012). ¿Educación cívica o educación para la ciudadanía? lo que acontece en Europa. En XIII Congreso Nacional de Educación Comparada. Huelva: Sociedad Española de Educación Comparada.

Rebollo Catalán, M. A., Vega Caro, L., y García Pérez, R. (2011). El profesorado en la aplicación de planes de igualdad: conflictos y discursos en el cambio educativo. RIE. Revista Investigación Educativa.

Rubio-Gil, Á., Martin-Pérez, A. M., Mesa-Olea, M. J., y Mesa-Olea, M. B. (2008). Influencias de las revistas juveniles en la sexualidad de las y los adolescentes. Madrid: Instituto de la mujer. Ministerio de Igualdad.

Tiana, A. (2011). Análisis de las competencias básicas como núcleo curricular en la educación obligatoria española. Bordón, 63(1), 63-75.

UNESCO. (2010). Orientaciones Técnicas Internacionales sobre Educación en Sexualidad: Un enfoque basado en evidencia orientado a escuelas, docentes y educadores de la salud. Paris.

Valero-Oltra, R., y López-Gosálbez, E. (2012). Modelos de intervención en sexología. Informació Psicológica, 103, 31-45.

Viñao, A. (1994). Sistemas educativos y espacios de poder: teorías, prácticas y usos de la descentralización en España. Revista Iberoamericana de Educación, 4, $29-64$.

VVAA. (2011). Educación para la sexualidad con bases científicas. Madrid.

\section{Referencias legislativas}

Ley 14/1970, de 4 de agosto, General de Educación y Financiamiento de la Reforma Educativa (1970). España.

Ley Orgánica 1/1990, de 3 de octubre, de Ordenación General del Sistema Educativo (1990). España.

Ley Orgánica 10/2002, de 23 de diciembre, de Calidad de la Educación (2002). España.

Ley Orgánica 2/2006, de 3 de mayo, de Educación (2006). España.

Ley Orgánica 8/1985, de 3 de julio, reguladora del Derecho a la Educación (1985). 
Educación sexual con enfoque de género en el currículo de la educación obligatoria en España: avances y situación actual Soraya Calvo González

Real Decreto 1006/1991 sobre Enseñanzas mínimas de la Educación Primaria (1991).

Real Decreto 1007/1991 sobre Enseñanzas mínimas de la Educación Secundaria Obligatoria (1991). España.

Real Decreto 1105/2014, de 26 de diciembre, por el que se establece el currículo básico de la Educación Secundaria Obligatoria y del Bachillerato (2014). España.

Real Decreto 126/2014, de 28 de febrero, por el que se establece el currículo básico de la Educación Primaria (2014). España.

Real Decreto 3087/1982, de 12 de noviembre, por el que se fijan las enseñanzas mínimas para el ciclo superior de Educación General Básica. (1982). España.

\section{Notas y/o Agradecimientos}

Esta investigación se ha llevado a cabo gracias a la financiación de la Fundación para el Fomento en Asturias de Investigación Científica Aplicada y la Tecnológica - FICYT (BP12128) y la Consejería de Educación del Principado de Asturias. 
